\title{
Condition monitoring of spar-type floating wind turbine drivetrain using statistical fault diagnosis
}

\author{
Ghane, Mahdi; Nejad, Amir Rasekhi; Blanke, Mogens; Gao, Zhen; Moan, Torgeir
}

Published in:
Wind Energy

Link to article, DOI:

10.1002/we.2179

Publication date:

2018

Document Version

Peer reviewed version

Link back to DTU Orbit

Citation (APA):

Ghane, M., Nejad, A. R., Blanke, M., Gao, Z., \& Moan, T. (2018). Condition monitoring of spar-type floating wind turbine drivetrain using statistical fault diagnosis. Wind Energy, 21(7), 575-589. https://doi.org/10.1002/we.2179

\section{General rights}

Copyright and moral rights for the publications made accessible in the public portal are retained by the authors and/or other copyright owners and it is a condition of accessing publications that users recognise and abide by the legal requirements associated with these rights.

- Users may download and print one copy of any publication from the public portal for the purpose of private study or research.

- You may not further distribute the material or use it for any profit-making activity or commercial gain

- You may freely distribute the URL identifying the publication in the public portal 


\title{
RESEARCH ARTICLE
}

\section{Condition monitoring of spar-type floating wind turbine drivetrain using statistical fault diagnosis}

\author{
Mahdi Ghane ${ }^{1,2}$, Amir Rasekhi Nejad ${ }^{1}$, Mogens Blanke ${ }^{2,3}$, Zhen Gao ${ }^{1}$ and Torgeir Moan ${ }^{1,2}$ \\ ${ }^{1}$ Center for Ships and Ocean Structures, Department of Marine Technology, Norwegian University of Science and Technology \\ (NTNU), NO-7491, Trondheim, Norway \\ ${ }^{2}$ Center for Autonomous Marine Operations and Systems (AMOS), Department of Marine Technology and Department of \\ Engineering Cybernetics, Norwegian University of Science and Technology (NTNU), NO-7491, Trondheim, Norway \\ ${ }^{3}$ Department of Electrical Engineering, Technical University of Denmark (DTU), DK 2800 Kgs. Lyngby, Denmark
}

\begin{abstract}
Operation and maintenance costs are significant for large-scale wind turbines, and particularly so for offshore. A wellorganized operation and maintenance strategy is vital to ensure the reliability, availability, and cost-effectiveness of a system. The ability to detect, isolate, estimate and perform prognoses on component degradation could become essential to reduce unplanned maintenance and downtime. Failures in gearbox components are in focus since they account for a large share of wind turbine (WT) downtime. This study considers detection and estimation of wear in the downwind main shaft bearing of a $5 \mathrm{MW}$ spar-type floating turbine. Using a high-fidelity gearbox model, we show how the downwind main bearing and nacelle axial accelerations can be used to evaluate the condition of the bearing. The paper shows how relative acceleration can be evaluated using statistical change detection methods to perform a reliable estimation of wear of the bearing. It is shown in the paper that the amplitude distribution of the residual accelerations follows a t-distribution and a change detection test is designed for the specific changes we observe when the main bearing becomes worn. The generalized likelihood ratio (GLR) test is extended to fit the particular distribution encountered in this problem, and closedform expressions are derived for shape and scale parameter estimation, which are indicators for wear and extent of wear in the bearing. The results in this paper show how the proposed approach can detect and estimate wear in the bearing according to desired probabilities of detection and false alarm.
\end{abstract}

Copyright $(0) 0000$ John Wiley \& Sons, Ltd.

\section{KEYWORDS}

Statistical Change Detection; Floating Wind Turbine; Wind Turbine Gearbox; Main Bearing; Fault Detection; Condition Monitoring

\section{Correspondence}

Centre for autonomous marine operations and systems (AMOS), Norwegian University of Science and Technology, NO-7491, Trondheim, Norway. E-mail: mahdi.ghane@ntnu.no

Received ... 


\section{INTRODUCTION}

1 Renewable energy can meet our ever-increasing need for energy. Among renewable resources, the use of wind energy 2 has increased spectacularly in recent decades [1]. Wind turbines (WTs) are exposed to highly variable and at times 3 severe environmental disturbances and, for floating turbines, also the fluctuating mechanical loads due to waves. These external factors contribute to large amount of downtime and high operation and maintenance (OM) costs during WT typical operational lifetime of 20 years [2]. OM costs are estimated to be $10-15 \%$ for onshore and $20-25 \%$ for offshore turbines of the total cost [3]. Thus, OM costs represent a substantial share of the total cost of energy for offshore wind [4].

Maintenance, in general terms, is classified into reactive (run to failure), preventive (periodic) and predictive maintenance (condition-based) [5, 6]. Efficient condition monitoring (CM) can greatly increase WT reliability and availability by decreasing the number of events where faults remain unnoticed and develop into failures [7]. Reviews of wind turbine condition monitoring techniques and challenges were presented in $[5,8,9]$. WT global response to fault and possibly failure of different subsystems has been thoroughly investigated in literature [10-12]. Some approaches are model-based, S. Cho et al. [13] showed a model-based fault detection for the blade pitch system, and Y. Qiu et al. [14] suggested a model-based approach to quantify gear fatigue life for wind turbine gearbox using supervisory control and SCADA data. Y. Qiu et al. [15] developed a synthesized thermal mode for wind turbine drivetrain fault diagnosis. Other methods are model-free, H. Niemann et al. [16] suggested a model-free diagnosis of rotor system fault.

Statistics show WTs undergo the largest amount of downtime related to drivetrain failures [17]. While a very few drivetrain full-scale dynamometer tests and field tests are available [18], numerical analysis and modeling play an important role for adaptation of condition monitoring techniques for WTs. Gearbox failures being initiated, in many cases, from bearings degradation $[19,20]$. The failure of the main bearings has significant impact on the gearbox life and on the turbine maintenance plan, in terms of the repair cost and long downtime. The gearbox is fundamentally designed to transfer the torque load, and is protected from non-torque loads, such as axial force and bending moments, by the main bearings. Once the main bearing damage occurs, the gearbox starts a fast degradation and eventual failure. Moreover, replacing the main bearing is often a very costly operation. Depending on the design type, it is often needed to dismantle the rotor and hub, which is a very costly operation especially for offshore WTs. Since model-based techniques can have high complexity and the modeling part can be a significant engineering effort, signal-based methods are prefered in industry. A signalbased frequency domain approach was proposed in [21], investigating the main shaft bearing fault detection based on absolute value principal component analysis technique. Hilbert spectral analysis was used to detect different faults in a 5 MW floating WT gearbox [22]. Statistical features of the signal in frequency domain, such as root-mean-square (RMS) of bearing fault frequencies, could also be used for prognosis. Frequency domain techniques are readily applicable to detection of wear, once it has developed, but these methods have difficulty in assessing gradual developments in wear and are difficult to use for prognosis. However, frequency domain analysis is more powerful to pinpoint the source of fault when dealing with several fault sources. It is, therefore, practically common to use the combined time-domain and frequency-domain analysis.

In this paper, statistical change detection is employed to estimate the magnitude of wear in the downwind main bearing, a spherical roller bearing. The relative axial motions between the main bearing and housing is scrutinized, and it is shown that relative acceleration follows a t-distribution with parameters that can describe the signal in the entire range from no wear to significant wear conditions. The paper shows how two t-distribution parameters, scale and shape, describe the development of damage, and a dedicated statistical test is developed that estimates damage through assessment of these parameters. The damage in this context is the worn condition - primarily due to fatigue - and initiation of fatigue cracks. Fatigue for rolling bearings is defined by ISO-15243 as the change in the structure, which is caused by the repeated stress developed in the contacts between the rolling elements and the raceways [23]. Such damage and degradation mechanism can be modelled by local stiffness reduction $[24,25]$ which has been employed in this paper.

A generalized likelihood ratio (GLR) test methodology is employed using closed form expressions for estimation of tdistribution parameters that are derived in the paper. Earlier results by the authors [26] showed that there was a correlation 
between wear and distribution, but did not assess the direct relation to wear, and did not consider a design with guaranteed diagnostic performance, both of which are essential to achieve reliable estimation and prognosis for wear. A salient feature of the suggested methodology is the ability to estimate both wear and the wear rate.

The remainder of the paper is organized as follows. Section 2 introduces the relevant modeling for WT and the drivetrain. Different fault scenarios and their classifications are discussed in Section 3. Section 4 derives the extension to the well known GLR test that makes it possible to estimate the degree of wear in the bearing, and Section 5 presents and discusses the results. Finally, conclusions are drawn in Section 6.

\section{WIND TURBINE AND DRIVETRAIN MODELS}

A 5 MW reference gearbox [27] mounted on the floating OC3 Hywind spar structure [28,29] was used in this study. This WT is a 3-bladed upwind WT with characteristic features shown in Table I. The spar-floating structure is column shaped and connected by mooring lines to the seabed. The spar structures have a large draft and a small waterline area. The details of the spar structure used in this paper were described by Nejad et al. [30].

Table I. Wind turbine specifications [28, 29].

\begin{tabular}{ll} 
Parameter & Value \\
\hline Type & Upwind/3 blades \\
Cut-in, rated and cut-out wind speed $(\mathrm{m} / \mathrm{s})$ & $3,11.4,25$ \\
Hub height $(\mathrm{m})$ & 87.6 \\
Rotor diameter $(\mathrm{m})$ & 126 \\
Hub diameter $(\mathrm{m})$ & 3 \\
Rotor mass $(\times 1,000 \mathrm{~kg})$ & 110 \\
Nacelle mass $(\times 1,000 \mathrm{~kg})$ & 240 \\
Hub mass $(\times 1,000 \mathrm{~kg})$ & 56.8 \\
\hline
\end{tabular}

The 5 MW reference gearbox used in this study was developed by Nejad et al. [27] for offshore WTs. The gearbox consists of three stages: two planetary and one parallel stage gears. Table II shows the general specifications of this gearbox. Figure 1 shows the gearbox and drivetrain layout. The gearbox topology is shown in Figure 2. The gearbox was designed with a 4-point support with two main bearings to reduce non-torque loads entering the gearbox.

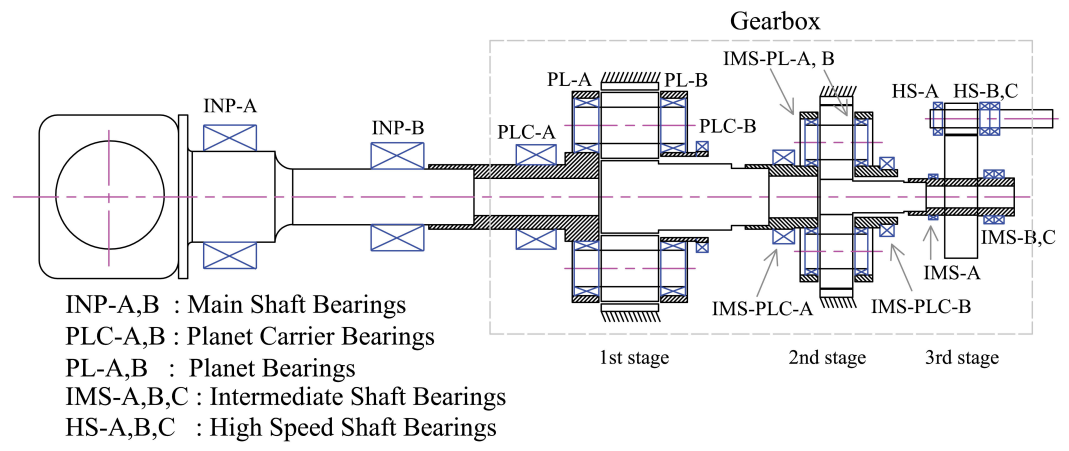

Figure 1. 5-MW reference gearbox layout [27].

In mutli-body simulation (MBS) model of this gearbox, the motions are applied on the bed plate and the external loads on the main shaft. The generator torque and speed are controlled on the generator side [27]. 
Table II. 5-MW reference gearbox specification [27].

\begin{tabular}{ll} 
Parameter & Value \\
\hline Type & 2 Planetary + 1 Parallel \\
1st stage ratio & $1: 3.947$ \\
2nd stage ratio & $1: 6.167$ \\
3rd stage ratio & $1: 3.958$ \\
Total ratio & $1: 96.354$ \\
Designed power $(\mathrm{kW})$ & 5000 \\
Rated input shaft speed $(\mathrm{rpm})$ & 12.1 \\
Rated generator shaft speed $(\mathrm{rpm})$ & 1165.9 \\
Rated input shaft torque $(\mathrm{kN} . \mathrm{m})$ & 3946 \\
Rated generator shaft torque $(\mathrm{kN} . \mathrm{m})$ & 40.953 \\
Total dry mass $(\times 1000 \mathrm{~kg})$ & 53 \\
Service life $($ year $)$ & 20 \\
\hline
\end{tabular}

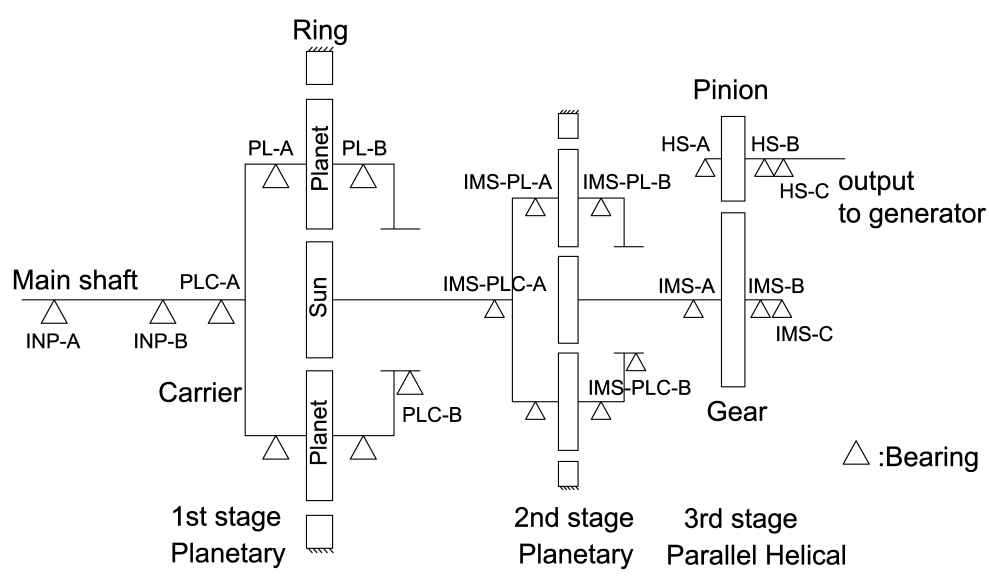

Figure 2. 5-MW reference gearbox topology [27].

\section{METHODOLOGY}

\subsection{De-coupled Approach \& Environmental Condition}

A decoupled approach was employed in this study for dynamic response analysis of the drivetrain. First, the forces and moments on the main shaft are obtained from the global response analysis. Second, they are used as inputs to a detailed gearbox MBS model in which simulations with a higher fidelity model and smaller time steps are performed.

The global analysis was conducted using an aero-hydro-servo-elastic code, SIMO-RIFLEX-AeroDyn [31]. Simulations were carried out at the rated wind speed with wave conditions characterized by a significant wave height $\mathrm{HS}=5 \mathrm{~m}$ and a peak period TP $=12 \mathrm{~s}$ (modeled by a JONSWAP spectrum). The turbulence intensity factor of the wind speed was taken to be 0.15 according to IEC 61400-1 [32]. The long-term environmental data used in this study were generated by a numerical hindcast model at the National and Kapodistrian University of Athens (NKUA) [33]. To minimize statistical uncertainties, six 3,800 s simulations were performed for all cases studied. The first $200 \mathrm{~s}$ of data were removed during post-processing to eliminate the transient effects associated with start-up.

In the MBS analysis, the bearings were modeled as force elements and obey force-deflection relations. The gears were modeled with compliance at the tooth and incorporate detailed tooth properties [27]. Earlier works on WT gearboxes based on the decoupled method include [34-36]. 


\subsection{Main Bearing Damage and Fault Cases}

As stated in Section 2, the $5 \mathrm{MW}$ reference drivetrain model consists of two main bearings. The second main bearing (INP-B) carries the axial force induced by the wind thrust force. The performance of this bearing is crucial to the gearbox life $[30,37]$. As this bearing undergoes degradation and wear, additional non-torque loads are transmitted to the gearbox and reduce the life of other components, particularly other bearings inside the gearbox. As highlighted in [20], most of the gearbox failures in WTs originate in the bearings. It is, therefore, important to monitor and evaluate the condition of this main bearing during operation.

In this study, the bearing was considered as one element whose lifetime can be modeled by the Lundberg-Palmgren equation [38], as specified by ISO 281 [39]. The life of roller bearings is limited to the fatigue life of the material from which they are made and is governed by the lubricant used. Extensive tests carried out by Lundberg and Palmgren in the SKF bearing manufacturing company in Sweden revealed that bearing life can be estimated directly from the applied loads [38]. Today, the Lundberg-Palmgren equations still form the basis of bearing selection and design and have been incorporated into the ISO 281 standard.

From the Lundberg-Palmgren hypothesis, the bearing life is expressed as follows [38-40]:

$$
L=\left(\frac{C}{P}\right)^{a}
$$

in which $L$ is the bearing basic life defined as the number of cycles that $90 \%$ of an identical group of bearings achieve under certain test conditions before fatigue damage appears, and $C$ is the basic load rating that is constant for a given bearing. The parameter $a=3$ for a ball bearing, and $a=\frac{10}{3}$ for roller bearings. The variable $P$ is the dynamic equivalent radial load calculated as follows:

$$
P=X F_{r}+Y F_{a}
$$

where $F_{a}$ and $F_{r}$ are the axial and radial loads on the bearing, respectively, and $X$ and $Y$ are constant factors obtained from the bearing manufacturer [39].

Equation (1) is a form of an SN curve formulation, which is used to estimate the fatigue damage on a bearing $[30,37,41,42]$.

The roller bearing contact is often modeled using the Hertzian contact theory [43]. In most bearing models, it is assumed that the bearings operate at moderate speeds, meaning that the effects of centrifugal, gyroscopic and frictional forces may be neglected and that the force on the rollers is expressed in the form of a load-deflection relationship [44-46]. This force is a function of the rollers material and hardness, the geometry and the applied load. If $\boldsymbol{q}$ represents the relative bearing deflection between the inner and outer races and $\boldsymbol{F}$ the applied forces and moments, the bearing stiffness matrix in nonlinear form may be expressed as follows [45]:

$$
\boldsymbol{K}=\frac{\partial \boldsymbol{F}}{\partial \boldsymbol{q}}
$$

A finite element method to calculate bearing stiffness was developed by [45]. Analytical methods for bearing stiffness calculation have also been published by Houpert [44]. New bearings often have relatively large stiffness values, in the order of $10^{8}$, and as the bearing wears and the surface hardness decreases, the contact zone increases, and the bearing stiffness decreases. Such decreases were observed in the experimental studies conducted by Qiu et al. [25].

This properties of a bearing provide a practical method for testing the damage detection methods using MBS models. Due to bearing complexities, bearings are modeled as a force element in an MBS model based on the bearing stiffness $[27,46]$. Bearing wear or damage can then be modeled by varying the stiffness matrix of the bearing, and the responses can be used for detection. This modeling approach has been used to model bearing faults in land-based wind turbines [47,48]. 
The remaining life of a worn bearing primarily depends on the load level at which the gearbox operates. The experimental tests conducted by Ocak et al. [49] on roller bearings indicate that the time from the initial observation of high vibrations until the ultimate failure can be as short as $10 \%$ of the total bearing life.

In this paper, the INP-B bearing, the downwind main shaft bearing Figure 2, wear is studied through monitoring the level of stiffness of the bearing for six different fault cases, as shown in Table III. The variable $K_{x}$ is the axial stiffness and $f$ and $n f$ represent the fault and non-fault cases, respectively. The use of the root mean square (r.m.s) value of the vibration signal is one of the methods used in the industry [50-52]; therefore, the vibration level of the INP-B bearing is exploited to explain the physical meaning of change of the bearing stiffness. According to ISO 20816-1 [52], the vibration velocity is often found to be sufficient for evaluating the severity of the vibration. ISO 20816-1 classifies four vibration zone boundaries based on the operational class of the machinery:

Zone A: new machines,

Zone B: acceptable zone for long-term operation,

Zone C: unacceptable for long-term operation,

Zone D: can cause severe damage.

The r.m.s values of the velocity for the INP-B bearing in different fault cases are calculated and evaluated based on the limits suggested by ISO 20816-1 [52] (see Table III). The jump in r.m.s observed for FC5 is due to a side band mesh frequency resonance at this fault case.

Table III. Velocity r.m.s of the INP-B in different fault cases.

\begin{tabular}{lllll}
\hline Fault case & $K_{x f}(\mathrm{~N} / \mathrm{m})$ & $\frac{K_{x f}}{K_{x n f}} \times 100 \%$ & r.m.s $(\mathrm{mm} / \mathrm{s})$ & vibration zone boundary \\
\hline FC0 & $4.06 \times 10^{8}$ & 100 & 0.8 & A/B \\
FC1 & $3.86 \times 10^{8}$ & 95 & 0.9 & A/B \\
FC2 & $3.45 \times 10^{8}$ & 85 & 0.9 & A/B \\
FC3 & $2.84 \times 10^{8}$ & 70 & 2.2 & B/C \\
FC4 & $2.03 \times 10^{8}$ & 50 & 2.4 & B/C \\
FC5 & $1.22 \times 10^{8}$ & 30 & 8.3 & C/D \\
FC6 & $4.06 \times 10^{7}$ & 10 & 7.7 & C/D \\
\hline
\end{tabular}

There are two different criteria to determine the critical level of stiffness (wear) of the INP-B bearing, the effect of the bearing wear on itself, represented by the level of vibration, and its degradation effect on other components. The wind turbine gearbox components usually are designed for the WT typical lifetime (20 years), and they are not designed to take the non-torque loads; hence, any source of non-torque loads play a critical role on the lifetime of the gearbox components. The main bearing, INP-B bearing, is the main part to protect the gearbox from non-torque loads. Thus, in addition to the INP-B vibrations, another appropriate indicator is the relative life of the bearings inside the gearboxfor instance, the planet carrier bearings in the first stage that carry the axial load. Based on the vulnerability map described by Nejad et al. [27,37], the PLC-B is selected for the life study. Table IV presents the remaining life of this bearing for different FCs. In this table, the remaining life is presented in normalized terms with respect to the nominal life, life at the FC0. The level of vibration at FC4 is acceptable for long-term operation; however, it is seen that FC4 (50\% reduction in INP-B stiffness) cause almost $70 \%$ reduction in PLC-B bearing lifetime. Accordingly, FC4 is selected as the critical fault case in this study.

Table IV. Fault cases \& PLC-B relative life.

\begin{tabular}{lll}
\hline Fualt cases & $\frac{K_{x f}}{K_{x n f}} \times 100 \%$ & PLC-B relative life \\
\hline FC0 & 100 & 100 \\
FC1 & 95 & 93 \\
FC2 & 85 & 78 \\
FC3 & 70 & 57 \\
FC4 & 50 & 33 \\
FC5 & 30 & 14 \\
FC6 & 10 & 3 \\
\hline
\end{tabular}




\section{STATISTICAL FAULT DIAGNOSIS}




$$
\begin{aligned}
H_{0}: p(z(i)) & \sim t\left(\mu_{0}, \sigma_{0}, \nu_{0}\right) \quad \text { for } \quad k-M+1 \leq i \leq k \\
H_{1}: p(z(i)) & \sim t\left(\mu_{0}, \sigma_{0}, \nu_{0}\right) \quad \text { for } \quad k-M+1 \leq i \leq k_{0}-1 \quad \text { and } \\
p(z(i)) & \sim t\left(\mu_{1}, \sigma_{1}, \nu_{1}\right) \quad \text { for } \quad k_{0} \leq i \leq k
\end{aligned}
$$

where the GLR test statistic, $g(k)$, and the univariate t-distribution are presented in equations (6) and (7), respectively:

$$
\begin{aligned}
& g(k)=\max _{k-M+1 \leq j \leq k} \underset{\sigma_{1}, \nu_{1}}{\operatorname{Sup}} \ln \frac{\prod_{i=k-M+1}^{k} f\left(z(i) ; \mu, \sigma_{1}, \nu_{1}\right)}{\prod_{i=k-M+1}^{k} f\left(z(i) ; \mu, \sigma_{0}, \nu_{0}\right)} \\
& f(z(i) ; \mu, \sigma, \nu)=\frac{\Gamma((1+\nu) / 2)}{\Gamma\left(\frac{\nu}{2}\right)(\pi \nu)^{0.5} \sigma} \times\left[\frac{\nu+\left(\frac{z(i)-\mu}{\sigma}\right)^{2}}{\nu}\right]^{-\frac{1+\nu}{2}}
\end{aligned}
$$

Furthermore, when an accurate estimation of the change time is not necessary, a cheaper approach from a computational point of view is possible by considering $M$ as a fixed-size moving window consisting of last $M$ samples. In this practical approach, the hypotheses change to equation (8), and the test statistics change from equations (6) to (9):

$$
\begin{gathered}
H_{0}: p(z(i)) \sim t\left(\mu, \sigma_{0}, \nu_{0}\right) \quad \text { for } \quad k-M+1 \leq i \leq k \\
H_{1}: p(z(i)) \sim t\left(\mu, \sigma_{1}, \nu_{1}\right) \quad \text { for } \quad k-M+1 \leq i \leq k \\
g(k)=\operatorname{Sup}_{\sigma_{1}, \nu_{1}} \ln \frac{\prod_{i=k-M+1}^{k} f\left(z(i) ; \mu, \sigma_{1}, \nu_{1}\right)}{\prod_{i=k-M+1}^{k} f\left(z(i) ; \mu, \sigma_{0}, \nu_{0}\right)}=\underset{\sigma_{1}, \nu_{1}}{\operatorname{Sup}} \ln G_{k-M+1}^{k}\left(\sigma_{1}, \nu_{1}\right)
\end{gathered}
$$

We note that if a change is detected by equation (9), at $k_{a}$, where the subindex $a$ stands for the alarm time, the only information about the change time, $k_{0}$, is that it lies over the interval of $k_{a}-M+1 \leq k_{0} \leq k_{a}$, which is acceptable in many industrial applications. However, it is also possible to estimate the change time, $k_{0}$, more accurately using the same approach as in equation (6). For each window size $M$ of data, change detection and estimation are performed simultaneously. The variable Sup $_{\sigma_{1}, \nu_{1}}$ represents the change magnitude estimation used to determine the best PDF fit to the new group of data, which gives the highest value for the test statistics. Generally, there are two approaches used to estimate the change magnitude, namely, the ME and the MLE [59]. Both approaches are addressed for the t-distribution in the next section.

\subsubsection{GLR test with Moment Estimator}

Different moments of the t-distribution are listed in Table V. The second and fourth moments can be used to estimate the change magnitudes, whereas $\sigma$ and $\nu$ are characteristic parameters.

Table V. The t-distribution moments [58]

\begin{tabular}{cccc}
\hline Mean & Variance & Skewness & Excess kurtosis \\
$E(x)=\mu$ & $\operatorname{Var}(x)=\sigma^{2} \frac{\nu}{\nu-2}$ & 0 & $\kappa=\frac{6}{\nu-4}$ \\
$\nu>2$ & & $\nu>4$ \\
\hline
\end{tabular}

Accordingly, $\hat{\sigma_{1}}$ and,$\hat{\nu_{1}}$ may be estimated using equations (10) and (11), where the subindex $M E$ indicates the moment estimator. 


$$
\hat{\nu}_{\mathrm{ME}}=\frac{6}{\left(\frac{1}{k-j+1} \sum_{i=j}^{k}\left(\frac{z_{i}-\mu}{\sigma}\right)^{2}\right)-3}+4
$$
determine a robust estimation.

\subsubsection{GLR test with maximum likelihood estimator}

The derivative with respect to $\sigma$ is:

Similarly, the derivative with respect to $\nu$ is:

$$
\hat{\sigma}_{\mathrm{ME}}=\left(\frac{1}{k-j+1} \sum_{i=j}^{k}\left(z_{i}-\mu\right)^{2}\right) \times \frac{\left(\hat{\nu}_{\mathrm{ME}}-2\right)}{\hat{\nu}_{\mathrm{ME}}}
$$

High order statistical moments are highly sensitive, as they are based on the tails of the distribution, where only a small percentage of the data carry useful information for high order moments. Consequently, a large window size is needed to

Maximum likelihood estimates of the two parameters, $\sigma_{1}$ and $\nu_{1}$ are found by equating $\left(\frac{\partial G_{k-M+1}^{k}\left(\sigma_{1}, \nu_{1}\right)}{\partial \sigma_{1}}\right)$ and $\left(\frac{\partial G_{k-M+1}^{k}\left(\sigma_{1}, \nu_{1}\right)}{\partial \nu_{1}}\right)$ to zero. The $G\left(\sigma_{1}, \nu_{1}\right)$ function, which is defined by equation (9), can be rewritten as:

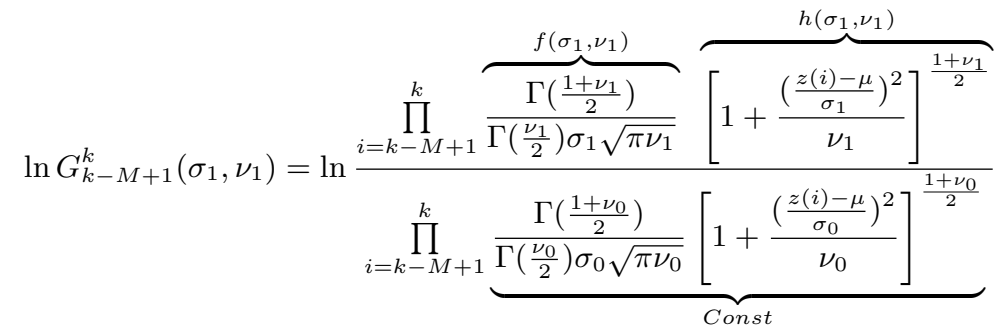

$$
\begin{aligned}
& \ln G_{k-M+1}^{k}\left(\sigma_{1}, \nu_{1}\right)=\sum_{i=k-M+1}^{k} \ln f\left(\sigma_{1}, \nu_{1}\right)-\left(\frac{1+\nu_{1}}{2}\right) \sum_{i=k-M+1}^{k} \ln h\left(\sigma_{1}, \nu_{1}\right)+\text { Const. }
\end{aligned}
$$

$$
\begin{aligned}
& \frac{\partial \ln G_{k-M+1}^{k}\left(\sigma_{1}, \nu_{1}\right)}{\partial \sigma_{1}}=\sum_{i=k-M+1}^{k}\left[\frac{\partial \ln f\left(\sigma_{1}, \nu_{1}\right)}{\partial \sigma_{1}}-\left(\frac{1+\nu_{1}}{2}\right) \frac{\partial \ln h\left(\sigma_{1}, \nu_{1}\right)}{\partial \sigma_{1}}\right] \\
& =\sum_{i=k-M+1}^{k}-\frac{1}{\sigma_{1}}+\frac{1+\nu_{1}}{\frac{\nu_{1} \sigma_{1}^{3}}{z_{i}^{2}}+\sigma_{1}}=\sum_{i=k-M+1}^{k} \frac{\nu_{1}}{\sigma_{1}} \times \frac{z_{i}^{2}-\sigma_{1}^{2}}{\sigma_{1}^{2} \nu_{1}+z_{i}^{2}} \\
& \Rightarrow \sum_{i=k-M+1}^{k} \frac{1}{\sigma_{1}^{2} \nu_{1}+z_{i}^{2}}=\frac{M}{\sigma_{1}\left(1+\nu_{1}\right)} .
\end{aligned}
$$

$$
\begin{gathered}
\frac{\partial \ln G_{k-M+1}^{k}\left(\sigma_{1}, \nu_{1}\right)}{\partial \nu_{1}}=\sum_{i=k-M+1}^{k} \frac{\partial}{\partial \nu_{1}}\left[\ln \frac{\Gamma\left(\frac{1+\nu_{1}}{2}\right)}{\Gamma\left(\frac{\nu_{1}}{2}\right) \sqrt{\pi \nu_{1}}}+\ln \frac{1}{\sigma_{1}}-\frac{1+\nu_{1}}{2} \ln \left(1+\frac{\left(\frac{z_{i}}{\sigma_{1}}\right)^{2}}{\nu_{1}}\right)+\text { Const }\right] \\
=\sum_{i=k-M+1}^{k} \frac{\partial}{\partial \nu_{1}}\left[\ln \Gamma\left(\frac{1+\nu_{1}}{2}\right)-\ln \Gamma\left(\frac{1+\nu_{1}}{2}\right)-\frac{1}{2} \ln \left(\pi \nu_{1}\right)-\ln \sigma_{1}-\frac{1+\nu_{1}}{2} \ln \left(1+\frac{\left(\frac{z_{i}}{\sigma_{1}}\right)^{2}}{\nu_{1}}\right)\right]
\end{gathered}
$$




$$
\begin{gathered}
\sum_{i=k-M+1}^{k} \frac{1}{2}\left[\psi\left(\frac{1+\nu_{1}}{2}\right)-\psi\left(\nu_{1}\right)-\frac{1}{2 \nu_{1}}-\ln \left(1+\frac{\left(\frac{z_{i}}{\sigma_{1}}\right)^{2}}{\nu_{1}}\right)+\left(\frac{1+\nu_{1}}{\nu_{1}}\right)\left(\frac{1}{1+\nu_{1}\left(\frac{\sigma_{1}}{z_{i}}\right)^{2}}\right)\right]=0 \\
\Rightarrow M \times\left\{\psi\left(\frac{1+\nu_{1}}{2}\right)-\psi\left(\frac{\nu_{1}}{2}\right)-\frac{1}{\nu_{1}}\right\}=\sum_{i=k-M+1}^{k}\left[\ln \left(1+\frac{\left(\frac{z_{i}}{\sigma_{1}}\right)^{2}}{\nu_{1}}\right)+\frac{\left(1+\nu_{1}^{-1}\right)}{\nu_{1}\left(\frac{\sigma_{1}}{z_{i}}\right)^{2}+1}\right],
\end{gathered}
$$

where $\psi(x)$ is the digamma function, which is the derivative of the gamma function:

$$
\psi(x)=\frac{d}{d x} \ln (\Gamma(x))=\Gamma^{\prime}(x) \Gamma^{-1}(x) .
$$

When $x$ is real and positive, which is the case here, the digamma function can be represented as:

$$
\psi(x)=\int_{0}^{\infty}\left(\frac{e^{-t}}{t}-\frac{e^{-x t}}{1-e^{-t}}\right) d t .
$$

A set of nonlinear equations (16) and (20) must be solved simultaneously to derive the MLE estimates of the $\sigma_{1}$ and $\nu_{1}$.

\subsection{GLR test statistics approximated by the Weibull distributions}

If the GLR test inputs are IID and Gaussian (or as an asymptotic property for infinite window size, $M \rightarrow \infty$ ), the distribution of the test statistics $\mathrm{g}(\mathrm{k})$ may be determined analytically and follows the Chi-squared distribution [53, 54]. This approach allows the determination of a threshold analytically based on desired values of probability of detection $P_{D}$ and probability of false alarm $P_{F}$. However, in many industrial applications, data are often correlated [55-57,60]. Thus, the actual distribution of the test statistics may be considerably different from the Chi-squared statistics obtained from the theory. One approach to address this issue is to approximate the test statistics from experimental data. Accordingly, several distributions were tested, the Weibull distribution was found to yield a good fit for the test statistics $g(k)$ [60].

\section{RESULTS AND DISCUSSION}

Based on the discussion in Section 3.2, FC4 is chosen as the critical wear case for the problem at hand, since this level of wear in the main shaft bearing significantly decreases the lifetime of the gearbox components. In FC4, the lifetime of the PLC-bearing is reduced by almost $70 \%$, although the vibration level of the main shaft bearing (INB-P) is still acceptable according to the ISO 20816-1 standard. Accordingly, two levels of wear are selected to evaluate the proposed diagnostic method, the FC1 (minimum wear) and the FC4 (critical wear). The nature of mechanical systems is such that appreciable displacements only occur at low frequencies, i.e., displacement gives the low frequency components most weight. Velocity tends to have reasonably uniform response from low to medium frequencies. Acceleration measurements gives more weight to the high frequency components and is, therefore, the logical choice for monitoring components that generate high frequency vibration such as bearings and gears.

Scrutinizing the power spectrum of relative displacement, velocity, and acceleration of the main shaft bearing and the nacelle, only relative acceleration does not have any appreciable components in wind and wave frequency range, meaning that the relative acceleration is more robust to input change of wind and waves and is relatively more sensitive to wear in the INP-B bearing. Thus, relative acceleration of the main shaft bearing and the nacelle is a well-suited measurement and is chosen to detect the wear in the bearing. The measured relative acceleration is obtained from two accelerometers. One mounted on the main shaft bearing and the other on the hub bed plate. Since the sensors noise are uncorrelated, by subtracting two measured accelerations the noise power will add up. However, the spectral noise parameter from commercial grade accelerometers are around $2 \times 10^{-6}\left(\mathrm{~m} / \mathrm{s}^{2}\right) / \sqrt{H z}$. The bandwidth of the accelerometer signals is below $50 \mathrm{~Hz}$, due to the low frequencies of wind/wave excitations, and the low rated rotational speed of the main shaft $(0.2$ $\mathrm{Hz}$ ). Therefore, the standard deviation of sensor noise would be about $2 \times 10^{-5}\left(\mathrm{~m} / \mathrm{s}^{2}\right)$ whereas the signal in no-fault case 
(lowest power), shown in Figure 3, is $0.08\left(\mathrm{~m} / \mathrm{s}^{2}\right)$ or 4000 times higher. Therefore, sensor noise is not relevant. Figure 3 depicts the relative acceleration time series and histogram for the FC0 (fault-free, red), FC1 (green) and FC4 (blue) levels.
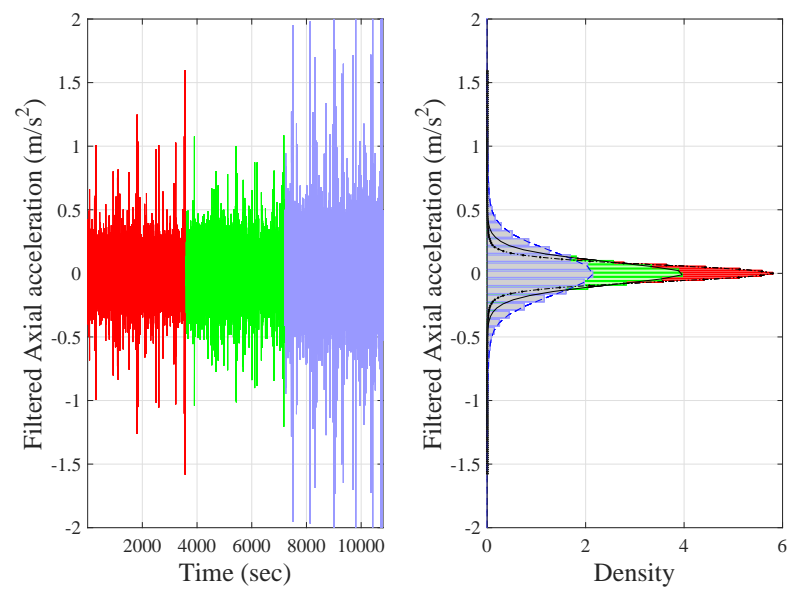

Figure 3. The time history and histogram of the relative axial acceleration for the FC0 (red), the FC1 (green) and the FC4 (blue)

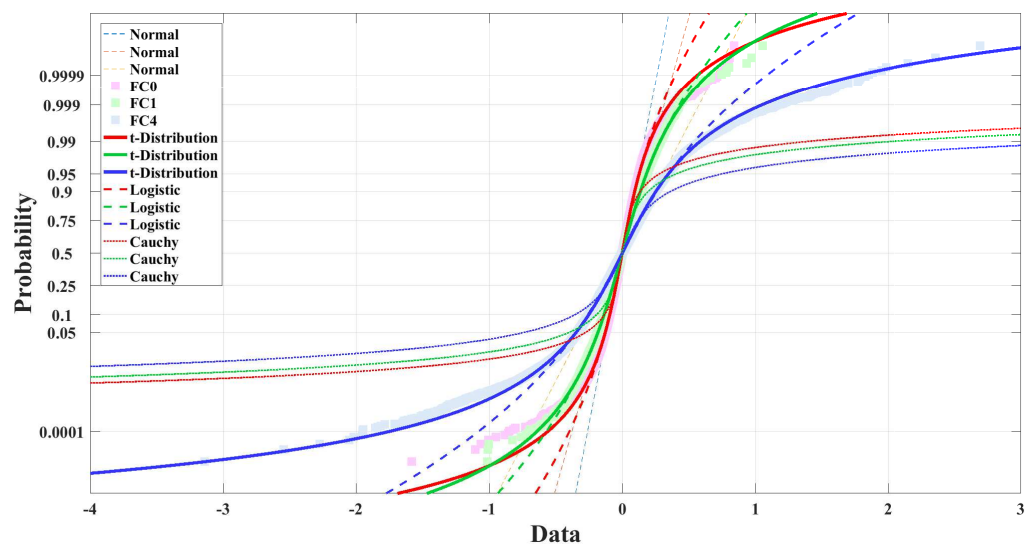

Figure 4. Normal probability plot and fitted distributions to FC0, FC1 and FC4 
theory (distribution model), whereas the Chi-square test might lack the required evidence for rejection [53]. Moreover, the Chi-square test statistic is sensitive to the bin width. A smaller bin width increases the noise effect, and a larger bin width causes a loss of information since the data are replaced by the median of the bins during the calculation of the test statistics, and unfortunately, there is no analytic expression for the optimal bin width.

Fortunately, the EDF-based GoF test statistics may still be modified and computed using several methods, such as the bootstrap method. Bootstrap resampling is conceptually simple but computationally expensive. In this approach, the critical values related to different significance levels may be estimated by bootstrap resampling of the original dataset, a data-based Monte Carlo method. The theory underlying the bootstrap method guarantees that the resulting significance levels are unbiased for a wide range of situations [62]. To save computational time, 10,000 samples are randomly selected from 720,000 samples (corresponding to a $1 \mathrm{~h}$ simulation time with a $200 \mathrm{~Hz}$ sampling rate) of relative axial acceleration to conduct the K-S test, as shown in Table VI. Parametric bootstrap resampling is also used to construct the critical values for each type of distribution and each fault case, as shown in Table VII.

Table VI. K-S goodness-of-fit values and p-values for different distributions for relative axial acceleration

\begin{tabular}{lcccccccc}
\hline Fault cases & \multicolumn{2}{c}{ Normal } & \multicolumn{2}{c}{ t-distribution } & \multicolumn{2}{c}{ Logistic } & \multicolumn{2}{c}{ Cauchy } \\
& K-S & p-value & K-S & p-value & K-S & p-value & K-S & p-value \\
\hline FC0 & 0.039 & 0.001 & 0.0067 & 0.154 & 0.0082 & 0.038 & 0.0636 & 0.001 \\
FC1 & 0.024 & 0.001 & 0.005 & 0.660 & 0.0067 & 0.212 & 0.0618 & 0.001 \\
FC2 & 0.015 & 0.001 & 0.0064 & 0.254 & 0.0121 & 0.001 & 0.0690 & 0.001 \\
FC3 & 0.01 & 0.031 & 0.0061 & 0.407 & 0.0145 & 0.001 & 0.0658 & 0.001 \\
FC4 & 0.048 & 0.001 & 0.0071 & 0.071 & 0.0087 & 0.022 & 0.06 & 0.001 \\
FC5 & 0.021 & 0.001 & 0.0071 & 0.125 & 0.0070 & 0.169 & 0.067 & 0.001 \\
FC6 & 0.014 & 0.001 & 0.0057 & 0.494 & 0.0143 & 0.001 & 0.0681 & 0.001 \\
\hline
\end{tabular}

There is always a deviation between the hypothesized distribution and the observed data (here simulation data). A GoF test may reject the hypothesized distribution if it is unlikely to observe such data given hypothesized distribution, i.e., the deviation is significantly high. The term significantly high can be interpreted using critical values $(c(\alpha))$ or $\mathrm{p}$-value. The K-S test accepts the hypothesized distribution if test statistics, maximum deviation between empirical cumulative distribution function and the hypothesized cumulative distribution function (D-value), is less than the critical values $(c(\alpha))$ for a given significance level $(\alpha)$. A p-value is the conditional probability of experiencing an extreme or higher deviation than the D-value on the condition that the data follows the hypothesized distribution. Similarly, the K-S test accepts the hypothesized distribution if the obtained $\mathrm{p}$-value is higher than the desired significance level $(\alpha)$, otherwise the K-S test rejects the hypothesized distribution.

Comparing the p-values and the K-S statistics with the critical values presented in Table VII show that the t-distribution is the best fit even for significance levels greater than $10 \%$ (probability level $90 \%=1-\alpha$ ). A low p-value (regarding the significance level) indicates that under a hypothesized distribution, it is unlikely that we will observe a D-value equal or higher than the observed value. The Logistic distribution is also acceptable at the $1 \%$ significance level. Therefore, the t-distribution is selected. The estimated t-distribution parameters for different fault cases are shown in Table VIII.

Table VII. Critical values resulted from bootstrap resampling

\begin{tabular}{lrrr}
\hline & \multicolumn{3}{c}{ Bootstrap Critical Values } \\
Distributions & $90 \%$ & $95 \%$ & $99 \%$ \\
\hline Normal & 0.0084 & 0.0091 & 0.0106 \\
t-distribution & 0.0074 & 0.0080 & 0.0093 \\
Logistic & 0.0074 & 0.0081 & 0.0093 \\
Cauchy & 0.0081 & 0.0089 & 0.0104
\end{tabular}


Table VIII. Estimated t-distribution parameters for different fault cases

\begin{tabular}{cccc}
\hline Fault cases & $\mu$ (location) & $\sigma($ scale parameter $)$ & $\nu$ (shape parameter $)$ \\
\hline FC0 & $7.903 \times 10^{-5}$ & 0.06395 & 5.45911 \\
FC1 & $3.255 \times 10^{-5}$ & 0.09694 & 7.64 \\
FC2 & $3.491 \times 10^{-5}$ & 0.07399 & 10.50 \\
FC3 & $-1.979 \times 10^{-7}$ & 0.23755 & 23.6446 \\
FC4 & $4.305 \times 10^{-5}$ & 0.1687 & 4.6897 \\
FC5 & $1.936 \times 10^{-5}$ & 0.6398 & 8.879 \\
FC6 & $2.627 \times 10^{-5}$ & 0.45298 & 14.3145 \\
\hline
\end{tabular}
bearing stiffness).

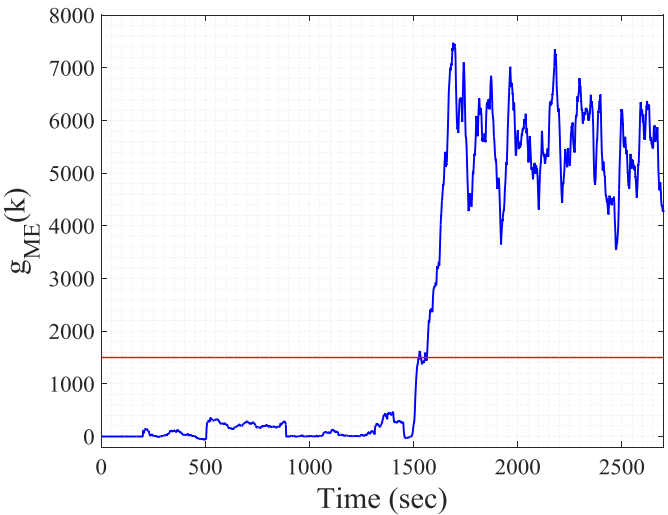

Figure 6. FC1 Decision function for $M 1=50000$ using moment estimator

Before fault occurrence (at $1500 \mathrm{~s}$ ), test statistics is close to zero while after a change it shoots up to a much higher positive value. The decision functions defer properly before and after fault occurrence, resulting in a robust detection. Different approaches (ME and MLE) were used to estimate the characteristic parameters, for a small window size $\left(M_{4}\right)$, the ME failed to estimate $\sigma$ and $\nu$ properly. A larger window size is hence needed, and the use of larger window size for both approaches (ME and MLE) provided almost identical results. The test statistics in Figures 5 and 6 result from assumed abrupt changes in the wear severity, whereas in practice bearings undergo wear gradually. Unfortunately, it is not possible to simulate gradual changes in wear from one fault case to another one using the SIMPACK software. However, the efficiency of the proposed method is tested on relatively small wear sizes, such as the FC1 level (i.e., a 5\% change in

The next step is to estimate the probability of detection and false alarm for the proposed method. It is too complicated to analytically derive an expression for the test statistics when the sample data are IID. Instead, the test statistics, $g_{M}(k)$, are approximated based on a long series of simulation data. The probability plot for the test statistics before and after fault occurrence for two window sizes (window size 1: 10,000 samples, $50 \mathrm{~s}$ and window size 2: 50,000 samples, $250 \mathrm{~s}$ ) are shown in Figures 7 and 8, respectively. The decision functions for the FC4 level properly roll out after the fault occurrence in both window sizes. However, for a smaller wear severities (FC1), 10,000 samples do not carry sufficient information to result in robust detection, as shown in Figure 7. Therefore, a larger sample size is required to ensure robust detection 
for the FC1, as shown in Figure Figure 8. The decision functions can be approximated using the Weibull distribution, as presented in equation (23). The related estimated parameters are shown in Table IX. The corresponding probabilities of detection and false alarm according to equations (25) and (24) are presented in Table X for the FC1 and FC4 levels.

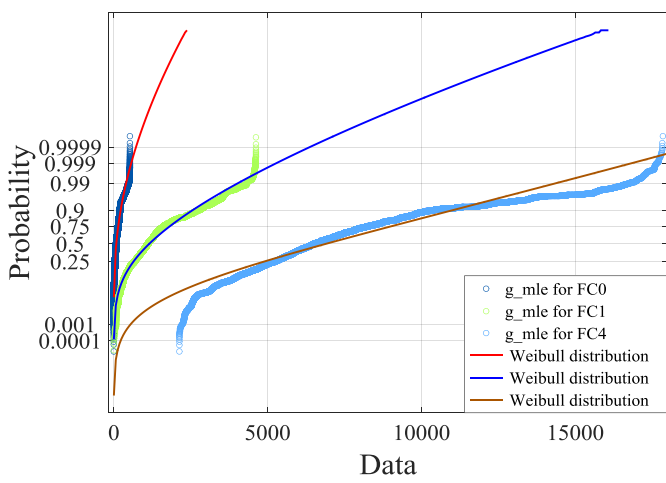

Figure 7. Test statistics $\mathrm{g}(\mathrm{k})$ for FCO, FC1 and FC4 for window size 1 equal to $50 \mathrm{~s}$ (10000 samples)

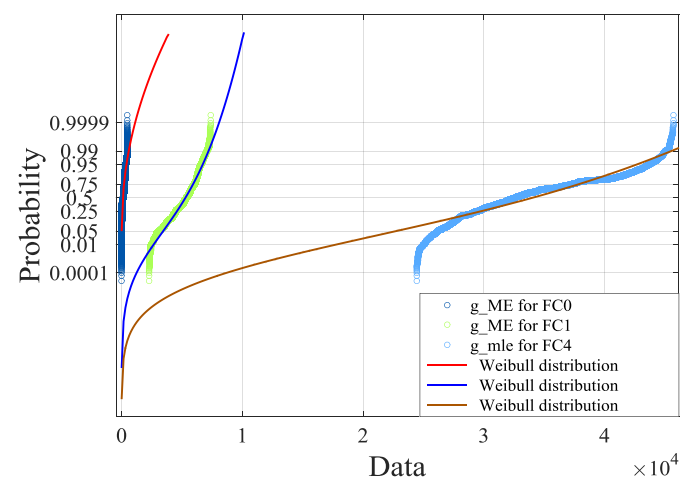

Figure 8. Test statistics $\mathrm{g}(\mathrm{k})$ for FC0, $\mathrm{FC} 1$ and FC4 for window size2 equal to $250 \mathrm{~s}$ ( 50000 samples)

$$
f(x \mid a, b)=\frac{b}{a}\left(\frac{x}{a}\right)^{b-1} \exp \left\{-\left(\frac{x}{a}\right)^{b}\right\}
$$

Table IX. Estimated Weibull distribution parameters for decision functions using different window sizes

\begin{tabular}{llll} 
& Hypothesis & $\hat{a}$ & $\hat{b}$ \\
\hline \multirow{2}{*}{ Window size1, 50 sec } & FC0 & 107.75 & 1.17 \\
& FC1 & 1462.16 & 1.51 \\
& FC4 & 7931.12 & 2.58 \\
Window size2, 250 sec & FC0 & 147.33 & 1.1 \\
& FC1 & 5608.38 & 6.10 \\
& FC4 & 35960.8 & 6.47
\end{tabular}

The selection of the detection threshold $h$ and the window size $(\mathrm{M})$ should be made based on our needs for the probability of false alarm $\left(P_{F}\right)$ and probability of detection $\left(P_{D}\right)$, as shown in Table $\mathrm{X}$. Since $P_{D}$ and $P_{F}$ are not independent, it is not possible to determine both as inputs at the same time. The test is designed to maximize the $P_{D}$ for a given $P_{F}$. The window sizes may be decreased until they indicate the same fitting distribution. Otherwise, important information may be lost. The first row of Table $\mathrm{X}$ shows that for small wear severity (FC1), 10,000 samples do not contain enough information, resulting in a relatively high probability of false alarm. The results for the other fault cases are satisfactory and can be improved by increasing the window sizes. The assumed window sizes (50 and $250 \mathrm{~s}$ ) are extremely short relative to the wear progress. Probabilities of detection and false alarm, presented in Table X, are estimated based on the distributions fitted to test statistics under $H_{0}$ and $H_{1}$. Figures 5 and 6 show that the test statistics exhibit a sharp roll off under $H_{1}$, while tails of the fitted distribution, here a Weibull distribution, cause underestimation of the detection probability of the test.

$$
P_{F}=P\left(g>h \mid \mathcal{H}_{0}\right)=\int_{h}^{\infty} p\left(g \mid \mathcal{H}_{0}\right) \mathrm{d} g \quad{ }^{313} \quad{ }^{312} \quad P_{D}=P\left(g \geq h \mid \mathcal{H}_{1}\right)=\int_{h}^{\infty} p\left(g \mid \mathcal{H}_{1}\right) \mathrm{d} g
$$


Table X. GLR test probabilities of detection and failure for different window sizes and for different thresholds

\begin{tabular}{lllll} 
window size & Fault cases & Threshold & $P_{D}$ & $P_{F}$ \\
\hline \multirow{2}{*}{50 sec (10000 samples) } & FC1 & 320 & 0.9040 & 0.0282 \\
& FC4 & 1400 & 0.9939 & $2.7577 \mathrm{e}-07$ \\
250 sec (50000 samples) & FC1 & 2200 & 0.9967 & $3.9687 \mathrm{e}-09$ \\
& FC4 & 7800 & 0.9999 & $2.3709 \mathrm{e}-34$ \\
\hline
\end{tabular}

The estimated parameters for $\sigma$ and $\nu$ at the FC0, FC1 and FC4 levels using the ME and MLE are depicted in Figure 9. The estimated characteristic parameters are suitably isolated for the cases FC0, FC1 and FC4. Although the estimation of $\nu$ is poor, the detection algorithm provides robust detection. Poor estimate of $\nu$ occurs because of the correlation between the data and the fact that $\nu$ determines the peakiness of the t-distribution, which is much more sensitive than the $\sigma$ value for a small data size. As an alternative approach, it is also possible to presume the $\nu$ value as its mean value depending on the problem. For instance, in this case $\nu=8$ is a sound assumption. Hence, $\sigma$ is the only value that needs to be estimated. Therefore, the computational cost is reduced at the expense of a small degradation in the probabilities of detection and failure.

Figure 9. Estimated t-distribution parameters for FC0, FC1 and FC4

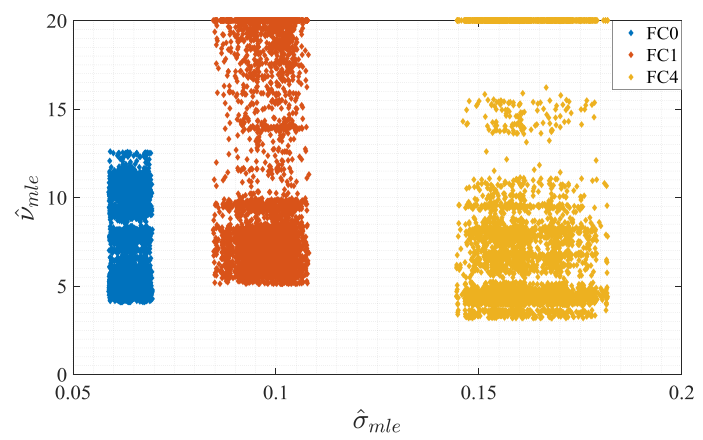

\section{CONCLUSIONS}

Wind Energ. 0000; 00:1-18 (c) 0000 John Wiley \& Sons, Ltd. 


\section{ACKNOWLEDGEMENT}

This work has been carried out at the Center for Autonomous Marine Operations and Systems (AMOS) and Center for Ships and Ocean Structures (CeSOS). The Norwegian Research Council is acknowledged as the main sponsor of AMOS and CeSOS. This work was supported by the Research Council of Norway through the Centers of Excellence funding scheme, Project numbers 146025-CeSOS and 223254-AMOS.

\section{REFERENCES}

1. Lauha Fried, Liming Qiao, Steve Sawyer, and Shruti Shukla. Global wind report. annual market update 2015. Technical report, Global Wind Energy Council, 2015.

2. Johan Ribrant. Reliability performance and maintenancea survey of failures in wind power systems. Master's thesis, Royal institute of technology (KTH), 2006.

3. Christopher A Walford. Wind turbine reliability: understanding and minimizing wind turbine operation and maintenance costs. United States. Department of Energy, 2006.

4. Bin $\mathrm{Lu}$, Yaoyu Li, Xin Wu, and Zhongzhou Yang. A review of recent advances in wind turbine condition monitoring and fault diagnosis. In Power Electronics and Machines in Wind Applications. PEMWA 2009. IEEE, pages 1-7. IEEE, 2009.

5. Pierre Tchakoua, René Wamkeue, Mohand Ouhrouche, Fouad Slaoui-Hasnaoui, Tommy Andy Tameghe, and Gabriel Ekemb. Wind turbine condition monitoring: State-of-the-art review, new trends, and future challenges. Energies, 7(4):2595-2630, 2014.

6. Alan Davies. Handbook of condition monitoring: techniques and methodology. Springer Science \& Business Media, 2012.

7. Eunshin Byon, Lewis Ntaimo, and Yu Ding. Optimal maintenance strategies for wind turbine systems under stochastic weather conditions. IEEE Transactions on Reliability, 59(2):393-404, 2010.

8. Wenxian Yang, Peter J Tavner, Christopher J Crabtree, Y Feng, and Y Qiu. Wind turbine condition monitoring: technical and commercial challenges. Wind Energy, 17(5):673-693, 2014.

9. Yanhui Feng, Yingning Qiu, Christopher J Crabtree, Hui Long, and Peter J Tavner. Monitoring wind turbine gearboxes. Wind Energy, 16(5):728-740, 2013.

10. Zhiyu Jiang, Madjid Karimirad, and Torgeir Moan. Dynamic response analysis of wind turbines under blade pitch system fault, grid loss, and shutdown events. Wind Energy, 17(9):1385-1409, 2014.

11. Zhiyu Jiang, Madjid Karimirad, Torgeir Moan, et al. Response analysis of parked spar-type wind turbine considering blade-pitch mechanism fault. International Journal of Offshore and Polar Engineering, 23(02), 2013.

12. Bachynski EE., Etemaddar M., Kvittem M., Luan C., and Moan T. Dynamic analysis of floating wind turbines during pitch actuator fault, grid loss, and shutdown. Energy Procedia, 35:210-222, 2013.

13. S Cho, Z Gao, and T Moan. Model-based fault detection of blade pitch system in floating wind turbines. In Journal of Physics: Conference Series, volume 753, page 092012. IOP Publishing, 2016.

14. Yingning Qiu, Lang Chen, Yanhui Feng, and Yili Xu. An approach of quantifying gear fatigue life for wind turbine gearboxes using supervisory control and data acquisition data. Energies, 10(8):1084, 2017.

15. Yingning Qiu, Yanhui Feng, Juan Sun, Wenxiu Zhang, and David Infield. Applying thermophysics for wind turbine drivetrain fault diagnosis using scada data. IET Renewable Power Generation, 10(5):661-668, 2016.

16. Henrik Niemann, Mahmood Mirzaei, Lars Christian Henriksen, and Niels Kjølstad Poulsen. Diagnosis of wind turbine rotor system. In American Control Conference (ACC), 2016, pages 3170-3175. IEEE, 2016.

17. Stefan Faulstich, Berthold Hahn, and Peter J Tavner. Wind turbine downtime and its importance for offshore deployment. Wind energy, 14(3):327-337, 2011. 
18. F Oyague, C Butterfield, and S Sheng. Gearbox reliability collaborative analysis round robin. National Renewable Energy Laboratory, Golden, CO, Report No. NREL/CP-500-45325, 2009.

19. F Spinato, PJ Tavner, GJW Van Bussel, and E Koutoulakos. Reliability of wind turbine subassemblies. IET Renewable Power Generation, 3(4):387-401, 2009.

20. Musial W., Butterfield S., and McNiff B. Improving wind turbine gearbox reliability. In European Wind Energy Conference, Milan, Italy, pages 7-10, 2007.

21. Moussa Hamadache and Dongik Lee. Wind turbine main bearing fault detection via shaft speed signal analysis under constant load. In Control, Automation and Systems (ICCAS), 2016 16th International Conference on, pages 1579-1584. IEEE, 2016.

22. Mahdi Ghane, Amir R Nejad, Mogens Blanke, Zhen Gao, and Torgeir Moan. Diagnostic monitoring of drivetrain in a 5 mw spar-type floating wind turbine using hilbert spectral analysis. Energy Procedia, 137:204-213, 2017.

23. ISO 281. Rolling bearings - damage and failures - terms, characteristics and causes, 2004.

24. Michael I Friswell and John ET Penny. Crack modeling for structural health monitoring. Structural Health Monitoring, 1(2):139-148, 2002.

25. Jing Qiu, Brij B Seth, Steven Y Liang, and Cheng Zhang. Damage mechanics approach for bearing lifetime prognostics. Mechanical systems and signal processing, 16(5):817-829, 2002.

26. Mahdi Ghane, Amir R Nejad, Mogens Blanke, Zhen Gao, and Torgeir Moan. Statistical fault diagnosis of wind turbine drivetrain applied to a 5mw floating wind turbine. In Journal of Physics: Conference Series, volume 753, page 052017. IOP Publishing, 2016.

27. A.R. Nejad, Y. Guo, Z. Gao, and T. Moan. Development of a 5 MW reference gearbox for offshore wind turbines. Wind Energy, 19(6):1089 - 1106, 2016.

28. Jonkman J., Butterfield S., Musial W., and Scott G. Definition of a 5-MW reference wind turbine for offshore system development. Technical Report NREL/TP-500-38060, National Renewable Energy Laboratory (NREL), 2009.

29. Jonkman J. Definition of the Floating System for Phase IV of OC3. National Renewable Energy Laboratory, 2010.

30. Nejad A.R., Bachynski EE., Kvittem M.I., Luan C., Gao Z., and Moan T. Stochastic Dynamic Load Effect and Fatigue Damage Analysis of Drivetrains in Land-based and TLP, Spar and Semi-Submersible Floating Wind Turbines. Marine Structures, 42:137-153, 2015.

31. Ormberg H. and Bachynski EE. Global analysis of floating wind turbines: Code development, model sensitivity and benchmark study. In 22nd International Ocean and Polar Engineering Conference, volume 1, pages 366-373, 2012.

32. IEC 61400-1. Wind turbines, part 1: Design requirements, 2005.

33. Lin Li, Zhen Gao, and Torgeir Moan. Joint distribution of environmental condition at five european offshore sites for design of combined wind and wave energy devices. Journal of Offshore Mechanics and Arctic Engineering, 137(3):031901-031901, 2015.

34. Xing Y., Karimirad M., and Moan T. Modelling and analysis of floating spar-type wind turbine drivetrain. Wind Energy, 17:565-587, 2014.

35. Nejad AR., Gao Z., and Moan T. On long-term fatigue damage and reliability analysis of gears under wind loads in offshore wind turbine drivetrains. International Journal of Fatigue, 61:116-128, 2014.

36. A.R. Nejad, Z. Jiang, Z. Gao, and T. Moan. Drivetrain load effects in a 5-MW bottom-fixed wind turbine under blade-pitch fault condition and emergency shutdown. Journal of Physics: Conference Series, 753(11):112011, 2016.

37. Nejad AR., Gao Z., and Moan T. Fatigue reliability-based inspection and maintenance planning of gearbox components in wind turbine drivetrains. Energy Procedia, 53:248-257, 2014.

38. Lundberg G. and Palmgren A. Dynamic capacity of roller bearings. Acta Polytechnica Mechanical Engineering Series, 2:5-32, 1952.

39. ISO 281. Rolling bearings - dynamic load ratings and rating life, 2007.

40. IEC 61400-4. Wind turbines, part 4: Standard for design and specification of gearboxes, 2012. 
41. Nejad A.R., Xing Y., Guo Y., Keller J., Gao Z., and Moan T. Effects of floating sun gear in a wind turbine's planetary gearbox with geometrical imperfections. Wind Energy, 18(12):2105-2120, 2015.

42. Nejad AR., Bachynski EE., Gao Z., and Moan T. Fatigue damage comparison of mechanical components in a landbased and a spar floating wind turbine. Procedia Engineering, 101:330-338, 2015.

43. Harris T.A. and Kotzalas M.N. Rolling bearing analysis. Taylor \& Francis, 5th edition, 2006.

44. Houpert L. A uniform analytical approach for ball and roller bearings calculations. Journal of tribology, 119(4):851858, 1997.

45. Yi Guo and Robert G. Parker. Stiffness matrix calculation of rolling element bearings using a finite element/contact mechanics model. Mechanism and Machine Theory, 51:32 - 45, 2012.

46. Jiang Z., Xing Y., Guo Y., Moan T., and Gao Z. Long-term contact fatigue analysis of a planetary bearing in a land-based wind turbine drivetrain. Wind Energy, doi:10.1002/we.1713, 2014.

47. Nejad AR., Odgaard PF., Gao Z., and Moan T. A prognostic method for fault detection in wind turbine drivetrains. Engineering Failure Analysis, 42:324-336, 2014.

48. Odgaard P.F. and Nejad A.R. Frequency based wind turbine gearbox fault detection applied to a $750 \mathrm{kw}$ wind turbine. In IEEE Conference on Control Applications (CCA), pages 1383-1388. IEEE, 2014.

49. Ocak H., Loparo K.A., and Discenzo F.M. Online tracking of bearing wear using wavelet packet decomposition and probabilistic modeling: A method for bearing prognostics. Journal of Sound and Vibration, 302(45):951 - 961, 2007.

50. Igba J., Alemzadeh K., Durugbo C., and Eiriksson E.T. Analysing rms and peak values of vibration signals for condition monitoring of wind turbine gearboxes. Renewable Energy, 91:90 - 106, 2016.

51. P Večeř, Marcel Kreidl, and R Šmíd. Condition indicators for gearbox condition monitoring systems. Acta Polytechnica, 45(6), 2005.

52. ISO 20816-1. Mechanical vibration - evaluation of machine vibration by measurements on non-rotating parts - part 1: general guidlines, 2016.

53. Mogens Blanke, Michel Kinnaert, Jan Lunze, Marcel Staroswiecki, and J Schrder. Diagnosis and fault-tolerant control. Springer-Verlag Berlin Heidelberg, 3rd edition, 2015.

54. Steven M Kay. Fundamentals of statistical signal processing: Detection theory,, volume v.2. Prentice Hall Upper Saddle River, NJ, USA:, 1998.

55. Roberto Galeazzi, Mogens Blanke, and Niels Kjølstad Poulsen. Early detection of parametric roll resonance on container ships. IEEE Transactions on Control Systems Technology, 21(2):489-503, 2013.

56. Soren Hansen and Mogens Blanke. Diagnosis of airspeed measurement faults for unmanned aerial vehicles. IEEE Transactions on Aerospace and Electronic Systems, 50(1):224-239, 2014.

57. Anders Willersrud, Mogens Blanke, Lars Imsland, and Alexey Pavlov. Drillstring washout diagnosis using friction estimation and statistical change detection. IEEE Transactions on Control Systems Technology, 23(5):1886-1900, 2015.

58. Samuel Kotz and Saralees Nadarajah. Multivariate t-distributions and their applications. Cambridge University Press, 2004.

59. Steven M Kay. Fundamentals of statistical signal processing. Prentice Hall PTR, 1993.

60. Mogens Blanke, Shaoji Fang, Roberto Galeazzi, and Bernt J Leira. Statistical change detection for diagnosis of buoyancy element defects on moored floating vessels. IFAC Proceedings Volumes, 45(20):462-467, 2012.

61. Michael A Stephens. Edf statistics for goodness of fit and some comparisons. Journal of the American statistical Association, 69(347):730-737, 1974.

62. G Jogesh Babu and CR Rao. Goodness-of-fit tests when parameters are estimated. Sankhyā: The Indian Journal of Statistics, 69:63-74, 2004.

63. Eric D Feigelson and G Jogesh Babu. Modern Statistical Methods for Astronomy: With R Applications. Cambridge University Press, 2012.

64. William Jay Conover. Practical nonparametric statistics. Wiley New York, 3rd edition edition, 1999. 